\title{
Measles outbreaks in the Emilia-Romagna Region, Italy, during 2016
}

\author{
Giulia Piccirilli, ${ }_{1}$ Angela Chiereghin, ${ }^{1}$ Gabriele Turello, ${ }^{1}$ Roberta Zuntini, ${ }^{2}$ Silvia Felici, ${ }^{1}$ Melissa Baggieri, ${ }^{3}$ \\ Loredana Nicoletti, ${ }^{3}$ Fabio Magurano, ${ }^{3}$ Gabriella Frasca, ${ }^{4}$ Maria Grazia Pascucci, ${ }^{4}$ Liliana Gabrielli, ${ }^{1}$ \\ Tiziana Lazzarotto ${ }^{1}$
}

${ }^{1}$ Microbiology Unit, Laboratory of Virology, Department of Specialized, Experimental, and Diagnostic Medicine, St. Orsola-Malpighi Polyclinic, University of Bologna; ${ }^{2}$ Unit of Medical Genetics, Department of Medical and Surgical Sciences, University of Bologna; ${ }^{3}$ WHO National Reference Laboratory for Measles and Rubella, Network of Subnational Reference Laboratory for Measles and Rubella, MoRoNet, Istituto Superiore di Sanità, Rome; ${ }^{4}$ Public Health Unit, Emilia-Romagna Region, Bologna, Italy

\begin{abstract}
Correspondence: Tiziana Lazzarotto, Microbiology Unit, Laboratory of Virology, Department of Specialized, Experimental, and Diagnostic Medicine, St. Orsola-Malpighi Polyclinic, University of Bologna, Via Massarenti 9, 40138 Bologna, Italy.

Tel.: +39.051.2143360 - Fax: +39 051.2143514.

E-mail: tiziana.lazzarotto@unibo.it

Key words: measles, molecular diagnosis, serological diagnosis, endemic MV strains.

Acknowledgements: the authors wish to acknowledge the technical support of Paola Bucci, Francesca Mazzilli and Antonella Marchi at the National Measles Reference Laboratory (ISS). We also would like to thank our Linguistic Consultant, Lucy Scioscia, for editing the English language text

Contributions: GP acquired and analyzed data as well as drafted the manuscript; AC, GT and SF contributed to the analysis and interpretation of data. RZ contributed to the sequence analysis. MB contributed to the phylogenetic analysis of MV strains detected. LN performed a critical revision of the manuscript. FM contributed to the phylogenetic analysis of MV strains detected and performed a critical revision of the manuscript. MGP and GF acquired epidemiological and clinical data. LG contributed to the analysis and interpretation of data and revision of manuscript. TL made substantial contributions to conception and design study, supervised the project, analyzed data and performed a critical revision of the manuscript.
\end{abstract}

Conflict of interest: the authors declare no potential conflict of interest.

Received for publication: 6 December 2017.

Accepted for publication: 7 December 2017.

(C) Copyright G. Piccirilli et al., 2017

Licensee PAGEPress, Italy

Microbiologia Medica 2017; 32:7224

doi:10.4081/mm.2017.7224

This article is distributed under the terms of the Creative Commons Attribution Noncommercial License (by-nc 4.0) which permits any noncommercial use, distribution, and reproduction in any medium, provided the original author(s) and source are credited. cases was reported during 2016. This study describes the measles outbreaks in Emilia-Romagna Region (ERR), one of the Italian regions mostly affected.

Materials and methods. A total of 101 suspected cases were reported in ERR during 2016. Laboratory diagnosis by serological and/or molecular methods was performed on 142 specimens (78 urine, 19 oral fluid and 45 sera) related to 97 suspected cases. For positive cases, measles virus (MV) strains involved were identified.

Results. Among 101 suspected cases, 72 (71.3\%) were confirmed. Vaccination status was known for 61 (84.7\%) cases, of which 56 (91.8\%) were unvaccinated. The highest incidence was found in the age group 15-39 years. In addition, for the $34.7 \%$ $(25 / 72)$ of confirmed cases, the transmission occurred in nosocomial settings, where healthcare workers were involved $(60 \%$ of cases). Roma/Sinti population were also involved in $12.5 \%(9 / 72)$ of confirmed cases. Both groups are considered hard-to-reach for immunization. The phylogenetic analysis showed circulation of MV strains belonging to genotype B3 and D8 in 45 (80.4\%) and 11 cases (19.6\%), respectively. In $94.7 \%$ of cases, the measles endemic transmission was demonstrated.

Conclusions. This data obtained through active surveillance showed the endemic transmission of MV within a population with immunity gaps including healthcare workers $(20.8 \%$ of confirmed cases), among which the spread of two endemic MV strains was observed.

\section{Introduction}

Measles virus (MV) is responsible for an infectious disease that often can result to serious complications $(3,20)$. The World Health Organization (WHO) set the goal to eliminate the indigenous MV transmission in all 53 Member States of the WHO European Region through the European Vaccine Action Plan 2015-2020 (5). Although progress has been made, 14 countries still remain endemic for measles transmission, including Italy, where many outbreaks have occurred in recent years $(1,19,21,26,27)$. During 2016, an increase in the reported measles cases in comparison to those recorded during 2015 (866 versus 259 cases) was observed (28). Six Italian regions were mostly affected, including the Emilia-Romagna Region (ERR) (28). In accordance with a national surveillance plan, physicians must report all clinically consistent measles/rubella cases to the Local Health Authorities (LHAs) that perform the epidemiological 
investigation and obtain specimens to send at designated laboratory for diagnosis and genotyping of MV/Rubella Virus (RV) $(11,19)$. In 2010, the Laboratory of Virology, St. Orsola-Malpighi Polyclinic, University of Bologna, was designated by the ERR authorities as Regional Reference Laboratory (LRR) for measles/rubella surveillance and in 2017 was accredited by National Reference Laboratory (NRL) in MoRoNet (Network of the Subnational Reference Laboratories), as LRR01 (18,21).

The study describes, through data obtained by an active surveillance system, the measles cases reported in ERR during 2016 focusing on cases reported in Bologna, the main city of the ERR. Moreover, the molecular characterization of MV circulated was performed comparing the strains identified with those detected in Italy and other European countries.

\section{Material and Methods}

\section{Study population}

From January to December 2016, 101 suspected measles cases were reported in ERR. A total of 142 clinical specimens (78 urine, 19 oral fluid and 45 sera) related to 97 suspected cases, were sent to LRR01 for laboratory diagnosis and genotyping of MV (Table 1). For each patient, personal data, vaccination status information, time of rash onset and the LHA where the case was reported, were provided in a patient information form sent with the specimens.

Furthermore, for cases reported in the LHA of Bologna, organized in 6 health districts (HDs), additional epidemiological and clinical data were obtained from the database of each HD (6). According to the 2012 European Commission case definition, the

Table 1. Characteristics of study population and clinical specimens.

\begin{tabular}{lc} 
Characteristics & No. \\
N. of suspected measles cases & 101 \\
Male/female & $52 / 49$ \\
\hline Median age & 30 years \\
& (range: 9 months - 80 years) \\
Vaccination status, n. & \\
1 dose & 9 \\
2 dose & 5 \\
Unvaccinated & 72 \\
Unknown & 15 \\
\hline LHA, n. & \\
Bologna & 35 \\
Cesena & 1 \\
Forlì & 2 \\
Imola & 3 \\
Ravenna & 5 \\
Modena & 2 \\
Parma & 30 \\
Piacenza & 8 \\
Reggio-Emilia & 3 \\
Rimini & 12 \\
Clinical specimens & \\
Urine/Oral fluid & 52 \\
Serum & 16 \\
Serum and Urine/Oral Fluid & 29 \\
NA & 4 \\
\hline
\end{tabular}

NA: not available. notified suspected cases were classified as confirmed, probable or possible (9).

\section{Laboratory methods}

\section{Serological test}

Serum specimens were tested by an automated chemiluminescence immunoassay, LIAISON ${ }^{\circledR}$ measles $\operatorname{IgM} / \mathrm{IgG}$ (DiaSorin, Italy), for the detection of MV-IgM and $\operatorname{IgG}$, following the manufacturer's instructions. According to the integrated surveillance program, the specimens that resulted negative for MV-IgM were tested for RV-IgM and IgG.

\section{Molecular test}

The detection of MV-RNA by PCR was performed on urine or oral fluid specimens. The extraction was carried out with the QIAsymphony-SP instrument (Qiagen, USA). Briefly, RNA was purified from $500 \mu \mathrm{L}$ of urine sediment or $800 \mu \mathrm{L}$ of oral fluid and eluted in $115 \mu \mathrm{L}$ and $140 \mu \mathrm{L}$, respectively. Reverse transcription of MV-RNA was performed as previously described (21). For each specimen, $4 \mu \mathrm{L}$ of cDNA were amplified by multiplex Real-Time PCR for the simultaneous detection of MV-RNA (114 bp, N gene) and RV-RNA (129 bp, P150 gene) using the set of primers and TaqMan probes described by Hubschen et al. (15).

\section{Sequencing and phylogenetic analysis}

For all MV-RNA positive specimens, the MV genome region required for genotype characterization was amplified and sequenced, as previously described (21). The sequences obtained were aligned by CLUSTAL-W and the genotype was assigned by comparison with WHO reference strains (24). Sequences, named according to official WHO nomenclature, were sent to NRL for the submission to MeaNs (the Measles Nucleotide Surveillance databank) (24). The phylogenetic trees were constructed by MEGA (version 7) using neighbor-joining method (22).

\section{Results}

\section{Laboratory confirmation}

Among the 101 suspected cases, 72 (71.3\%) were confirmed, $5(5 \%)$ were classified as possible, $3(3 \%)$ as probable and 21 (20.7\%) were discarded. The 72 confirmed cases were positive by serological and/or molecular methods. Particularly, 11/72 (15.3\%) cases were tested only for MV-IgM detection, 34/72 (47.2\%) only by molecular method and 27/72 (37.5\%) by both methods. Among these, discordant results were obtained in 6/27 (22.2\%) cases. One case was negative for MV-IgM and positive for MV-RNA detection. The specimens (serum and urine) were collected within 24 hours after onset of rash. In the remaining 5 cases, positive results were obtained by serological diagnosis and negative by molecular methods. These specimens were collected in a median time equal to 12 days (range: 4-24 days) after onset of rash. In 56/72 (77.7\%) cases, MV-RNA was identified in urine/oral fluid specimens and genotyping of MV strains was performed.

\section{Vaccination status, age distribution and complications of confirmed measles cases.}

The majority of suspected measles cases was reported during the first 5 months of 2016 (Figure 1). Among the 72 confirmed cases, vaccination status was known for 61 (84.7\%): $56(91.8 \%)$ 
were unvaccinated and $5(8.2 \%)$ vaccinated with 1 dose. The median age of confirmed cases was 29 years (range: 9 months-68 years) and the highest incidence was found in the age group 15-39 years (Figure 2). Complications were reported in 48/72 (66.6\%) cases (Table 2); the most frequent were diarrhea and hepatitis.

Table 2. Complications reported in the confirmed measles cases.

\begin{tabular}{lc} 
Complication & No. (\%) \\
Diarrhea & $8(16.7)$ \\
Hepatitis & $8(16.7)$ \\
\hline Keratoconjunctivitis & $7(14.6)$ \\
Pneumonia & $6(12.5)$ \\
\hline Encephalitis & $5(10.4)$ \\
Stomatitis & $4(8.3)$ \\
\hline Others* & $10(20.8)$ \\
Total & 48 \\
\hline
\end{tabular}

*Others: persistent hyperpyrexia $(\mathrm{n}=1)$, Laryngotracheobronchitis $(\mathrm{n}=6)$, seizure $(\mathrm{n}=3)$.

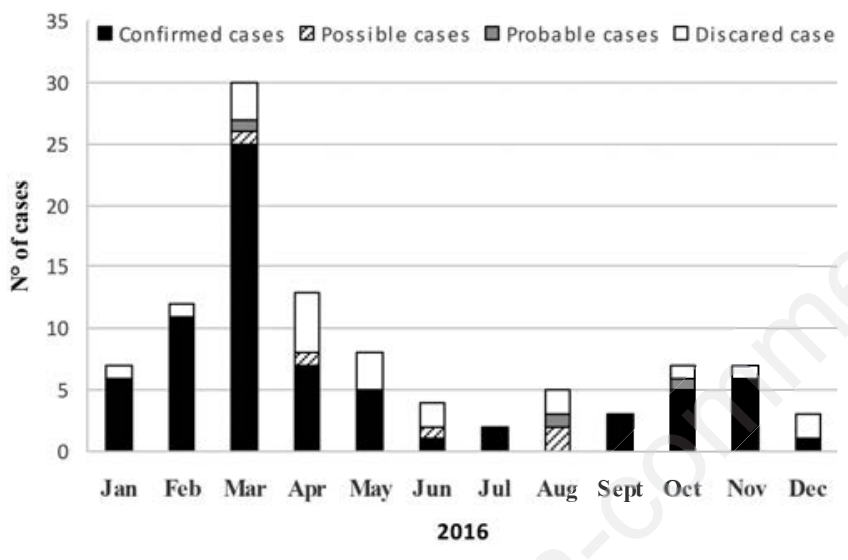

Figure 1. Suspected measles cases distributed by month of rash onset during 2016.

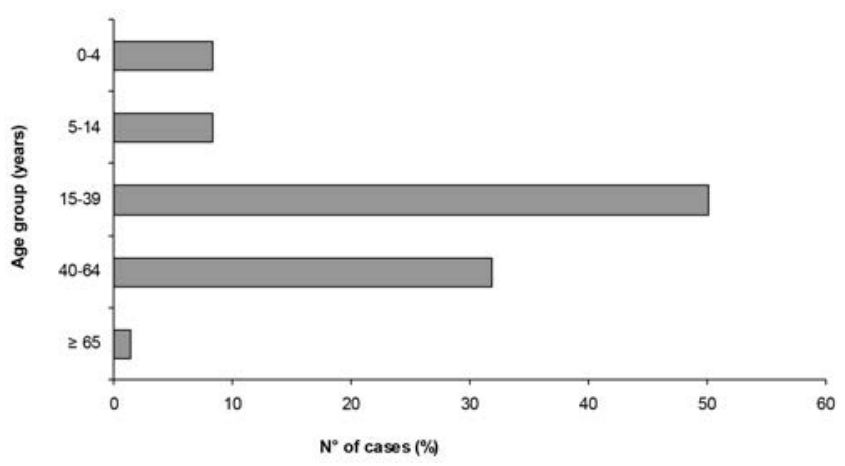

Figure 2. Measles confirmed cases distributed by age groups.

\section{MV strains identified in ERR outbreaks}

Phylogenetic analysis showed two genotypes of MV circulated during 2016: B3 was found in $45(80.4 \%)$ cases and D8 in the remaining $11(19.6 \%)$.

Sequences belonging to genotype B3 clustered into three main variants. (Figure 3). These variants show a $100 \%$ identity with three different WHO named strains. The strain MVs/Como.ITA/ 32.15/ was found in 26 cases. Among these, 3 were sporadic cases identified in the LHAs of Reggio-Emilia and

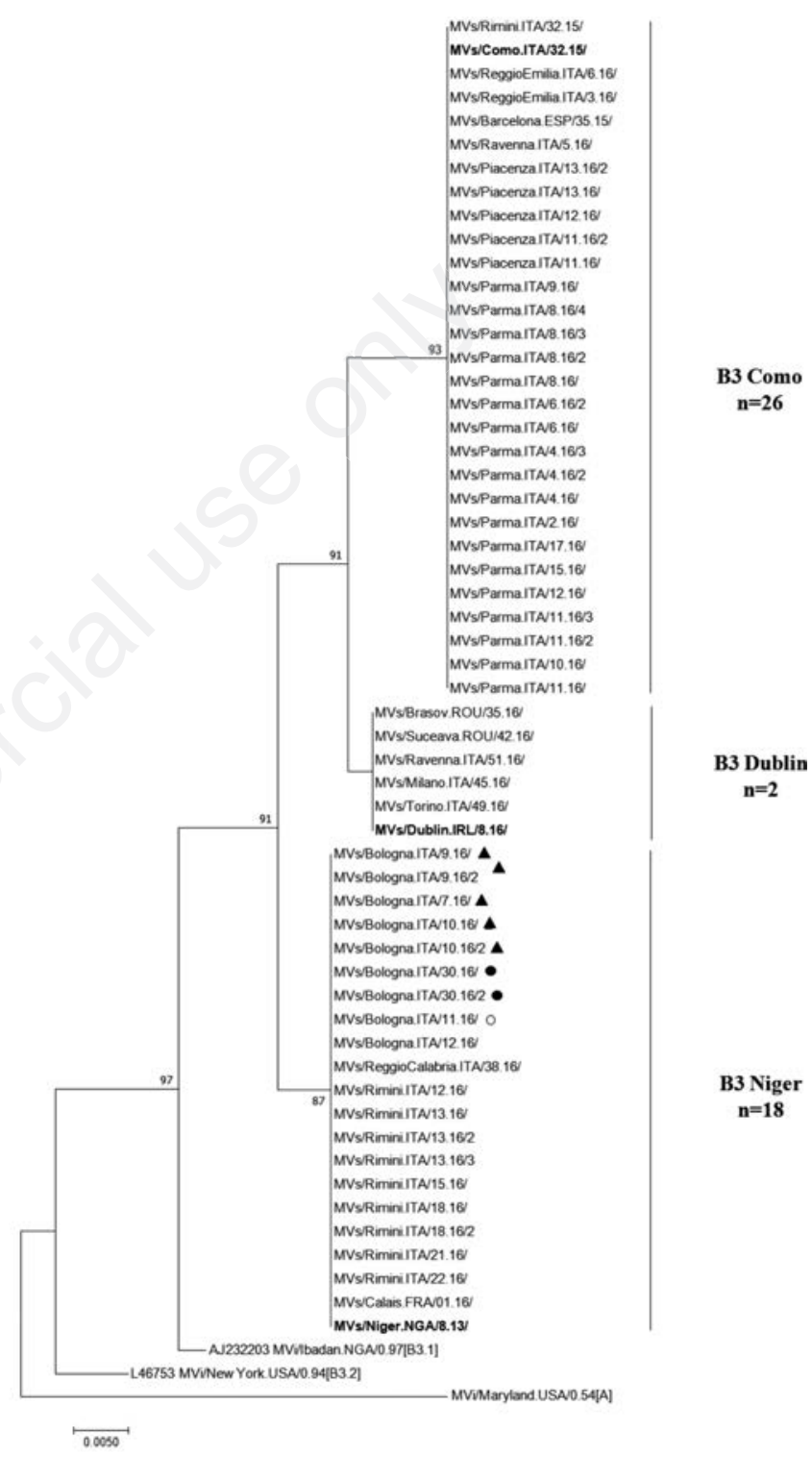

Neighbour-joining tree for nucleotide sequences of MV strains belonging to genotype B3. Sequences from Bologna clusters: $(\boldsymbol{\Delta})$ cluster $1,(\bullet)$ cluster $3,(\circ)$ cluster 2 . In brackets are reported the number of identical sequences detected in ERR during 2016. The evolutionary history was inferred by using the Maximum Likelihood method based on the Kimura 2-parameter model. The tree is drawn to scale, with branch lengths measured in the number of substitutions per site. Bootstrap values $>80 \%$ are reported. Evolutionary analyses were conducted in MEGA7.

Figure 3. Phylogenetic tree showing MV stains belonging to genotype $\mathbf{B} 3$. 
Ravenna, while 23 were correlated with an outbreak that occurred from November 2015 to April 2016 in two northern Italian regions, ERR and Lombardy. Of note, during this outbreak, a total of 24 cases were confirmed in ERR (the MV strain was not available for one case confirmed only by serological method), and in $62.5 \%(15 / 24)$ of cases, the transmission occurred in the nosocomial settings, where healthcare workers (HCWs) were involved (12/15 cases, 80\%) (14).

The second named strain MVs/Dublin.IRL/8.16/, was found in a single imported case from Romania. In fact, the viral genome sequence had $100 \%$ identity with strain that circulated during the same period in Romania (MVs/Brasov.ROU/35.16, MVs/Suceava.ROU/42.16). This strain has continued to circulate in Italy during 2017 (data not shown). The third strain, identified as $\mathrm{MVs} / \mathrm{Niger} . \mathrm{NGA} / 8.13 /$, circulated in ERR and other Italian regions, was identified in 18 confirmed cases reported in the LHAs of Bologna ( $n=9$, see below) and Rimini $(n=9)$. Among the cases confirmed in LHA of Rimini, the 55.5\% (5/9) were from the Roma/Sinti population.

For the genotype D8, 4 MV strains were identified. In particular, the sequences found in 7 cases reported in the LHA of Bologna (see below) showed $100 \%$ identity with the named strain MVs/Osaka.JPN/29.15/, the same that circulated in Italy and in Europe (Figure 4). Two cases were imported from the UK (MVs/Parma.ITA/35.16) and Denmark (MVs/ReggioEmilia. ITA/15.16) and showed $100 \%$ identity each other and with the named strain MVs/Cambridge.GBR/5.16. Other one was identified in a patient returning from Austria and belongs to the named variant
MVs/Victoria.AUS/6.11; the last was identified in a patient returning from India. The nucleotide analysis showed 100\% identity between these latter two sequences and MV strains circulated in visited countries.

\section{Measles clusters identified in the LHA of Bologna}

During 2016, 35 suspected measles cases were reported. Five measles clusters were identified in the 6 HDs of the LHA of Bologna (Figure 5), in which confirmed $(n=18)$, probable $(n=3)$ and possible cases $(\mathrm{n}=2)$ were involved. In the remaining 16 suspected cases, 6 were classified as sporadic (in 3 cases endemic MV strains were identified), 1 case as imported and 5 were discarded. The first cluster, n.1 (February-March 2016), originated from an unvaccinated infant (18 months old) who was admitted at a local Emergency Department (ED), where the infection was then transmitted to 4 unvaccinated people: 2 infants and 2 adults. Of these, 1 infant and 1 adult were members of the Roma/Sinti community and they transmitted, outside of hospital, the infection to one unvaccinated adult relative. The other infected child was readmitted to the ED where the MV infection was transmitted to two unvaccinated infants. In this cluster, one case was classified as probable, while the remaining 7 were confirmed. The results of genotyping analysis were available for 5 cases, in which the Niger variant of genotype B3 was identified (Figure 3). The identical sequence was detected in another cluster, n. 2 (during March), originated from an unvaccinated adult who transmitted the infection to a family member (a 35 month old child). One case was confirmed and the other classified as possible. The same

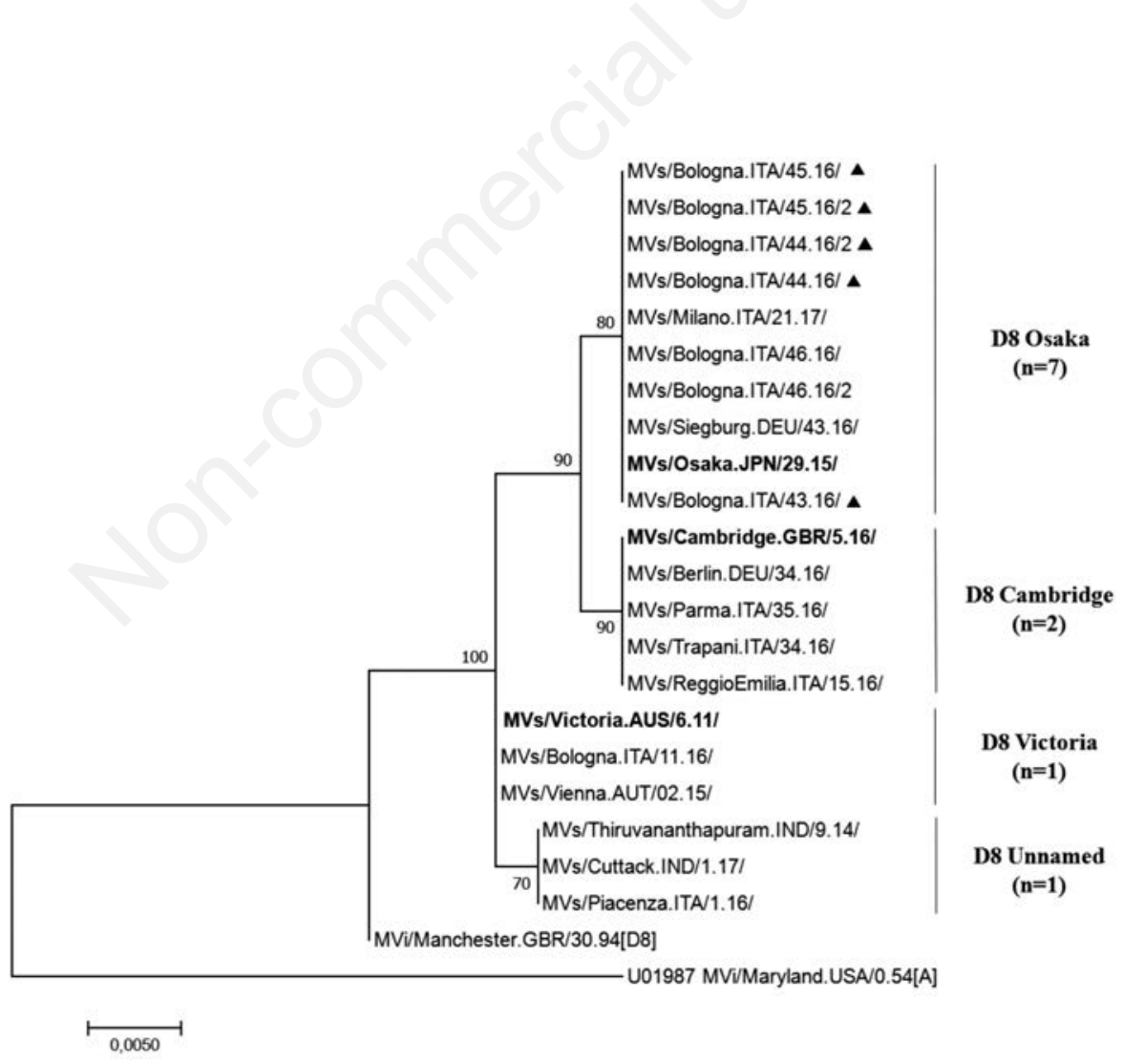

Neighbour-joining tree for nucleotide sequences of MV strains belonging to genotype D8. Sequences identified in the Bologna cluster 5 ( $\boldsymbol{\Delta}$ ). In brackets are reported the number of identical sequences detected in ERR during 2016. The evolutionary history was inferred by using the Maximum Likelihood method based on the Kimura 2-parameter model. The tree is drawn to scale, with branch lengths measured in the number of substitutions per site. Bootstrap values $>70 \%$ are reported. Evolutionary analyses were conducted in MEGA7.

Figure 4. Phylogenetic tree showing MV stains belonging to genotype D8. 
variant was also detected in cluster n. 3 (during July) involving 2 adult siblings (31 and 33 years old). Both showed clinical measles symptoms, after a trip to Calabria (Southern Italian region) where they had contact with a confirmed measles case. Phylogenetic analysis identified the Niger variant of genotype B3, circulating in ERR as well as in Calabria (Figure 3). An additional family cluster, n.4 (during August), affected three unvaccinated siblings. The index case was a 15 year old boy returning from the UK. He transmitted the infection to two siblings (12 and 9 years old). The laboratory diagnosis and molecular characterization of MV strain involved was not performed because their parents refused biological specimen collection. Therefore, the index case was classified as a probable imported case and the siblings as possible cases. The last cluster, n.5 (during November), originated from an unvaccinated student (17 years old) that was admitted to a local ED for persistent hyperpyrexia and onset of rash. In the nosocomial setting, 2 adult patients and 3 nurses had direct contact with the index case and developed measles symptoms after 10-15 days. Two of these nurses transmitted the infection to 2 unvaccinated adults outside the hospital. A total of 8 confirmed cases were involved. Results of sequence analysis were available in 5 cases and identified an Italian endemic MV strain of genotype D8 (Figure 4). Among the 18 confirmed measles cases reported in the LHA of Bologna, transmission occurred in nosocomial setting for 10 cases $(55.5 \%)$ and in $3 / 10$ $(30 \%)$ cases were involved HCWs. As control measures carried out to limit the spread of infection, contacts of confirmed cases were invited to be vaccinated. The measles immunity status of HCWs was verified and MRR vaccine immunization was offered to susceptible individuals.

\section{Discussion}

During 2016, a high number of measles cases was reported in the ERR with incidence equal to $1.8 / 100,000$ inhabitants (17). The $71.3 \%$ of suspected cases were confirmed by laboratory diagnosis using molecular and/or serological methods. Among the cases tested by both methods, discordant results were obtained in $22.2 \%$ of cases due to the timing of specimen collection $(4,16,21,23$,). In the $77.8 \%$ of confirmed cases the phylogenetic analysis was performed and results showed the circulation of MV strains belonging to genotype B3 $(80.4 \%)$ and D8 $(19.6 \%)$. The data reflect the nationwide $\mathrm{MV}$ genotypes circulation during last two years $(12,19,25)$. Three strains of B3 and four of D8 were identified. In $5.4 \%$ of cases, strains imported from other countries were found. In the remaining $94.6 \%$ of cases, MV strains circulating in Italy were identified, showing the endemic transmission of virus within a population with immunity gaps. In fact, in the ERR, as in other Italian regions, the MMR vaccine coverage was below the target of $95 \%$, which was set to eliminate the endemic transmission of MV (2). During 2015, in ERR, the coverage for the second dose of MMR was equal to $88.9 \%$ in 7 year old children (7). Among the confirmed cases, for which vaccination status was known, $91.8 \%$ were unvaccinated and most of the cases were reported among adults ranging between ages of 15 to 39 . As described by other authors, in Italy, in this group the higher number of susceptible individuals is present (19).

Finally, our data also showed the spread of MV in a nosocomial setting, occurring in $34.7 \%(25 / 72)$ of confirmed cases. Of note, HCWs were involved in $60 \%(15 / 25)$ of cases, suggesting that

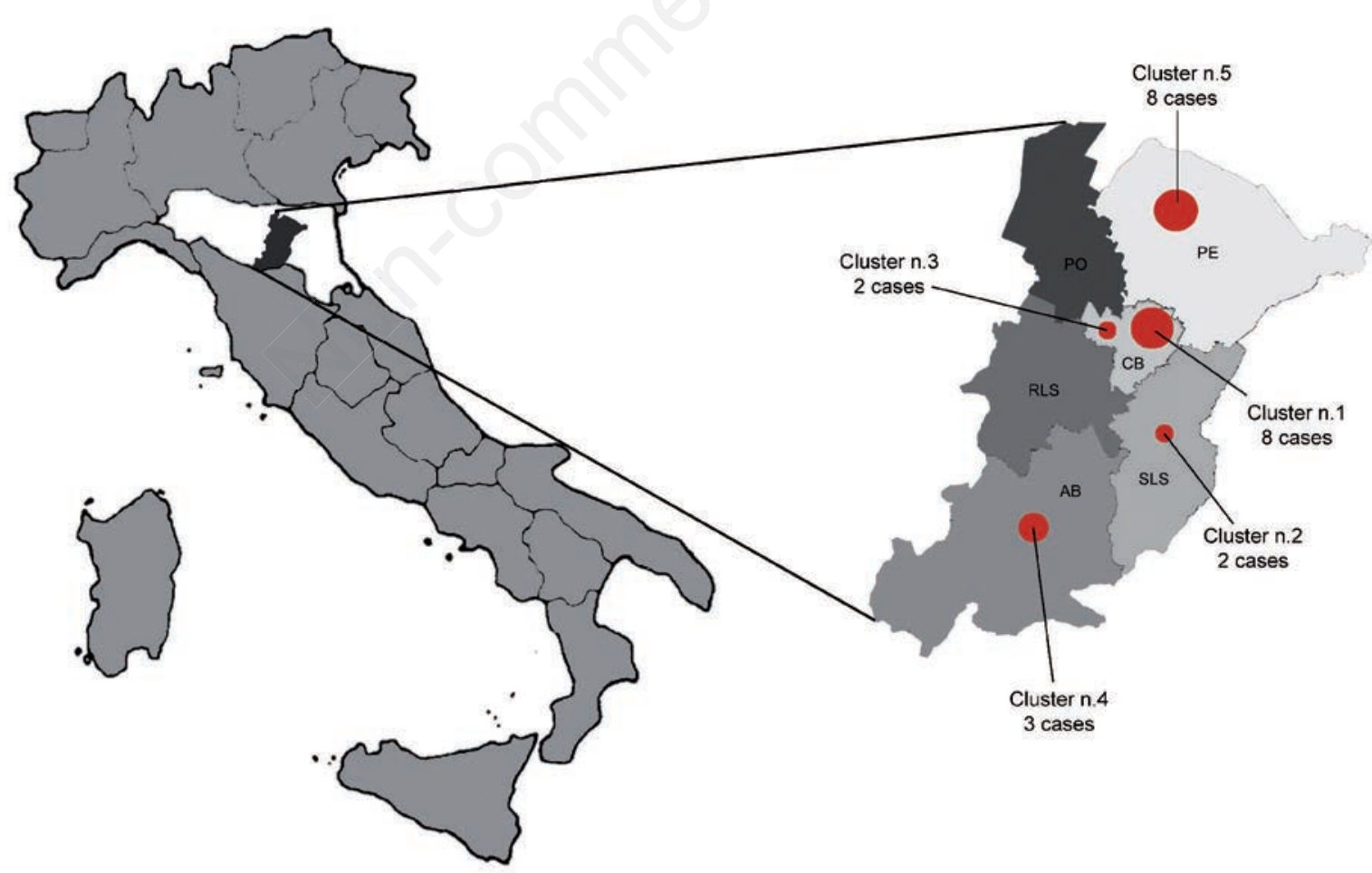

HDs of LHA of Bologna: Appennino bolognese (AB), Città di Bologna (CB), Pianura Est (PE), Pianura Ovest (PO), Reno-Lavino-Samoggia (RLS) and San Lazzaro di Savena (SLS). Clusters are represented with red circle areas proportional to the total number of reported measles cases.

Figure 5. Distribution of the measles clusters identified in the LHA of Bologna during 2016. 
measles immunization rate remains low among hospital staff, as described by other authors $(10,12,13,14)$. In fact, this group is considered to be hard-to-reach by immunization programmes, as well as the Roma/Sinti population that were also involved in the $12.5 \%$ $(9 / 72)$ of confirmed cases $(8,22)$. The lower vaccination coverage rates in hard-to-reach populations, contribute to the spread of MV and therefore pose a public health concern $(8,10,13,14)$. It is important to underline that, in ERR, the transmission of measles among HCWs occurred in 20.8\% (15/72) of confirmed cases. In fact, $\mathrm{HCW}$ s have a higher risk to measles infection than the general population given their greater opportunity for exposure and being a source of infection for their collogues and susceptible patients, including those hospitalised with critical conditions $(10,13,14)$.

\section{Conclusions}

In conclusion, MV continues to circulate in ERR and in Italy due to insufficient vaccine coverage. In Italy, much progress has been made through laboratory confirmation and molecular characterization of MV strains that help to identify the sources of virus and allow prompt planning and effective implementation of control measures. In particular, the successful establishment of a National Laboratory Network (MoRoNet) has led to high quality standards in laboratory surveillance. Nevertheless, further efforts will be needed to eliminate the endemic MV transmission, such as improving the vaccine coverage and implementing the immunization activities for all under-vaccinated population groups.

\section{References}

1. Amendola A, Bubba L, Piralla A, et al. Surveillance and vaccination coverage of measles and rubella in Northern Italy. Hum Vaccin Immunother 2015;11:206-13.

2. Bonanni P, Ferro A, Guerra R, et al. Vaccine coverage in Italy and assessment of the 2012-2014 National Immunization Prevention Plan. Epidemiol Prev 2015;39:146-58.

3. Buchanan R, Bonthius DJ. Measles virus and associated central nervous system sequelae. Semin Pediatr Neurol 2012;19:107-14.

4. Center for Disease Control and Prevention. Manual for the Surveillance of Vaccine-Preventable Diseases. Measles. Available from: https://www.cdc.gov/vaccines/pubs/surv-manual/chpt07-measles.pdf

5. Datta SS, O'Connor PM, Jankovic D, et al. Progress and challenges in measles and rubella elimination in the WHO European Region. Vaccine 2017; Available from: https://doi.org/10.1016/j.vaccine.2017.06.042.

6. Emilia-Romagna Region. Emilia-Romagna Regional Health Service Facilities, expenditure, activities as of 31.12.2013 report on 4 years of activity (2010-2013). 2015. Available from: http://salute.regione.emilia-romagna.it/documentazione/rapporti/ssr/201cthe-emilia-romagna-regional-healthservice-facilities-expenditure-activities-as-of-31-12-2013report-on-4-years-of-activity-2010-2013-201d/view

7. Emilia-Romagna Region. Coperture vaccinali nell'infanzia e nell'adolescenza. Anno 2015. 2016. Available from: http://www.epicentro.iss.it/temi/vaccinazioni/pdf/Coperture \% 20 vaccinali $\% 20$ nell'infanzia $\% 20 \mathrm{e} \% 20$ nell' adolescenza $\% 20$ $\% 20$ aggiornamento\%20anno\%202015.pdf

8. European Centre for Disease Prevention and Control. Review of outbreaks and barriers to MMR vaccination coverage among hard-to-reach populations in Europe. Available from: https://ecdc.europa.eu/sites/portal/files/media/en/publications/Publications/MMR-vaccinationhard-to-reach-population-review-2013.pdf

9. European Commission. Commission Implementing Decision of 08 August 2012 (2012/506/EU) amending Decision 2002/253/EC laying down case definitions for reporting communicable diseases to the Community network under Decision No 2119/98/EC of the European Parliament and of the Council. Official Journal of the European Union 27.9.2012; L262/1. Available from: http://eur-lex.europa.eu/legal-content/EN/TXT/PDF/?uri=CELEX:32012D0506\&from=EN

10. Fiebelkorn AP, Seward JF, Orenstein WA. A global perspective of vaccination of healthcare personnel against measles: systematic review. Vaccine 2014;32:4823-39.

11. Filia A, Tavilla A, Bella A, et al. Measles in Italy, July 2009 to September 2010. Eurosurveillance 2011; 16: pii=19925. Available from: http://www.eurosurveillance.org/ViewArticle. aspx?ArticleId=19925

12. Filia A, Amendola A, Faccini $M$, et al. Outbreak of a new measles B3 variant in the Roma/Sinti population with transmission in the nosocomial setting, Italy, November 2015 to April 2016. Euro Surveill. 2016;21: pii $=30235$. Available from: http://dx.doi.org/10.2807/1560-7917.ES.2016.21.20.30235

13. Fortunato F, Tafuri S, Cozza V, et al. Low vaccination coverage among Italian healthcare workers in 2013. Hum Vaccin Immunother 2015;11:133-9.

14. Haviari S, Bénet T, Saadatian-Elahi M, et al. Vaccination of healthcare workers: a review. Hum Vaccin Immunother 2015;11:2522-37.

15. Hübschen JM, Kremer JR, De Landtsheer S, et al. A multiplex TaqMan PCR assay for the detection of measles and rubella virus. J Virol Methods 2008;149:246-50.

16. Hummel KB, Lowe L, Bellini WJ, et al. Development of quantitative gene-specific real-time RT-PCR assays for the detection of measles virus in clinical specimens. J Virol Methods 2006;132:166-73.

17. Istituto Superiore di Sanità. Morbillo \& Rosolia News. Rapporto $\mathrm{N}^{\circ} 34$-2017. 2017. Available from: http://www.epicentro.iss.it/ problemi/morbillo/bollettino/RM_News_2016_34.pdf

18. Istituto Superiore di Sanità. Mo.Ro.NET: la Rete Nazionale di Laboratori di Riferimento per il Morbillo e la Rosolia. 2017. Available from: http://moronetlab.it/index.php/convegno/

19. Magurano F, Baggieri M, Filia A, et al. Towards measles elimination in Italy: Virological surveillance and genotypes trend (2013-2015). Virus Res 2017;236:24-9.

20. Piccirilli G, Lazzarotto T, Chiereghin A, et al. Spotlight on measles in Italy: why outbreaks of a vaccine-preventable infection continue in the 21 st century. Expert Rev Anti Infect Ther 2015;13:355-62.

21. Piccirilli G, Chiereghin A, Pascucci MG, et al. Molecular detection and genetic characterization of circulating measles virus in northern Italy. J Clin Virol 2016;81:34-42.

22. Kumar S, Stecher G, Tamura K. MEGA7: molecular evolutionary genetics analysis version 7.0 for Bigger Datasets. Mol Biol Evol 2016;33:1870-4.

23. Warrener L, Slibinskas R, Chua KB, et al. A point-of-care test for measles diagnosis: detection of measles-specific IgM antibodies and viral nucleic acid Bull World Health Organ 2011;89:652-82.

24. World Health Organization. Measles virus nomenclature update: 2012. Wkly Epidemiol Rec 2012;87:73-81.

25. World Health Organization. Measles and Rubella Surveillance 
Data. Distribution of genotypes in 2015. Available from: http://www.who.int/immunization/monitoring_surveillance/fu 11_measles_genotype_map_Jan15_Dec15.jpg?ua=1

26. World Health Organization. Measles in the WHO European Region. Fact sheet, July 2016. Available from: http://www.euro.who.int/_data/assets/pdf_file/0007/276379/ Factsheet-Measles-en.pdf?ua $=1$

27. World Health Organization. Measles outbreaks across Europe threaten progress towards elimination. 2017. Available from: http://www.euro.who.int/en/media-centre/sections/pressreleases/2017/measles-outbreaks-across-europe-threatenprogress-towards-elimination

28. World Health Organization. A report on the epidemiology of selected vaccine-preventable diseases in the European Region. 2017. Available from:http://www.euro.who.int/_data/assets/ pdf_file/0009/337464/EpiBrief_1_2017_EN.pdf 